Voix et Images

voixetimages

\title{
Ce que deviennent les « classiques »
}

\section{Robert Dion}

Volume 33, numéro 1 (97), automne 2007

Michel Marc Bouchard

URI : https://id.erudit.org/iderudit/017538ar

DOI : https://doi.org/10.7202/017538ar

Aller au sommaire du numéro

Éditeur(s)

Université du Québec à Montréal

ISSN

0318-9201 (imprimé)

1705-933X (numérique)

Découvrir la revue

Citer ce compte rendu

Dion, R. (2007). Compte rendu de [Ce que deviennent les « classiques »]. Voix et Images, 33(1), 170-175. https://doi.org/10.7202/017538ar

Ce document est protégé par la loi sur le droit d'auteur. L'utilisation des services d'Érudit (y compris la reproduction) est assujettie à sa politique d'utilisation que vous pouvez consulter en ligne.

https://apropos.erudit.org/fr/usagers/politique-dutilisation/
Cet article est diffusé et préservé par Érudit.

Érudit est un consortium interuniversitaire sans but lucratif composé de l’Université de Montréal, l’Université Laval et l'Université du Québec à Montréal. Il a pour mission la promotion et la valorisation de la recherche. https://www.erudit.org/fr/ 


\author{
R E C H E R C H E \\ Ce que deviennent les «classiques» \\ $+++$ \\ ROBERT DION \\ Université du Québec à Montréal
}

Malgré ce qui peut les différencier de prime abord, mes lectures du mois dernier - un ouvrage sur la traduction littéraire tiré d'une thèse de doctorat et un collectif publié dans une collection entièrement vouée à l'une de nos auteures cardinales m'ont fait songer au processus d'institutionnalisation accélérée de la littérature québécoise depuis les deux ou trois dernières décennies. Non que ce mouvement n'ait pas été perceptible auparavant, mais il semble, ces derniers temps, s'être précipité, et la recherche - c'est ce qui m'est apparu avec plus de force que jamais - n'est pas en reste en fait d'accréditations et de consécrations. Même quand elle paraît se limiter au simple constat du phénomène d'institutionnalisation, elle ne manque pas, en effet, par le choix de ses objets et plus encore par ses conclusions, de participer à la tendance générale. On n'est pas forcé d'être agacé, mais il y a des moments où, oui, l'on souhaiterait un peu plus de distance...

Je n'ai encore jamais parlé des Cahiers Anne Hébert, qui en sont pourtant à leur septième livraison avec ce dossier ayant pour titre Filiations. Anne Hébert et Hector de Saint-Denys Garneau ${ }^{1}$. On ne fait que commencer, au Québec, à publier des Cahiers dédiés à nos icônes nationales, et l'on peut raisonnablement penser que bientôt il y aura pléthore de "Sociétés des amis...» de tel ou tel de nos écrivains fétiches. C'est sans doute une bonne nouvelle pour les aficionados, pour le loisir littéraire en général et peut-être pour l'industrie touristique; pour la recherche, ça reste à voir. Avouerai-je que ce dossier ne m'a qu'à moitié convaincu? Il y a certes du savoir dans ces pages, mais aussi beaucoup de dévotion, et quelque chose d'un ronron critique qui ne sert pas nécessairement l'œuvre d'Anne Hébert. Je ne suis pas un spécialiste, je veux bien l'admettre, ni même un admirateur inconditionnel de l'écrivaine: j'ai toujours trouvé à ses romans quelque chose de vieillot sous les

1 Nathalie Watteyne (dir), Filiations. Anne Hébert et Hector de Saint-Denys Garneau, Sherbrooke/Montréal, Centre Anne-Hébert-Université de Sherbrooke/Fides, 2007. 
procédés modernes (trop) éprouvés. Je ne suis donc pas le mieux placé pour juger de la critique hébertienne telle qu'elle se déploie ici; mais il m'apparaît tout de même que, plus d'un lustre après la mort de l'auteure, il serait temps de soumettre son œuvre à des interrogations plus osées, ou simplement de la lire un peu moins dans le sens du poil. À mon sens, certaines études hors dossier, tout particulièrement celles qui portent sur les avatars de la maternité (Stéphanie Viau) et sur les spécificités de la prose poétique hébertienne (Adela Gligor), ne constituent pas des avancées très notables par rapport à ce qu'on disait déjà d'Anne Hébert à l'époque de mes études universitaires, dans les lointaines années 1980. Quant à la troisième contribution hors dossier, d'Annabelle $\mathrm{M}$. Rea à propos de la fonction du vêtement dans Un habit de lumière, elle traite certes d'un sujet moins convenu et d'un roman qui n'a pas, jusqu'à présent, obtenu toute l'attention critique qu'il méritait, mais elle reste à la surface, justement, et ne lève pas vraiment le voile, si je puis me permettre de filer la métaphore, sur les dessous du texte...

Les articles du dossier ${ }^{2}$, pour leur part, abordent de plus ou moins près ces filiations qu'annonce l'intitulé des Cahiers. À côté des très anecdotiques contributions de Benoît Lacroix (un témoignage assez ténu sur la vie au manoir des Garneau), de Frédéric Brochu (sur la généalogie des Hébert et des Taché) et de Marie-Andrée Lamontagne (un aperçu de la biographie d'Anne Hébert qu'elle est à écrire), le lecteur aura deux morceaux plus substantiels à se mettre sous la dent. Il y a d'abord la contribution de Karim Larose qui retrace le passage, opéré par Saint-Denys Garneau, d'une poésie comme chant, c'est-à-dire comme vision lyrique de l'écriture, à une poésie comme parole, en deçà ou au-delà du lyrisme, comme voix des choses, comme mots vécus. Ce nouveau paradigme qu'inaugure le poète, surtout dans la partie la plus confidentielle de son œuvre, c'est, dit Larose, sa cousine Anne Hébert qui, dans son essai «Poésie, solitude rompue», lui donnera son envol au tournant des années 1960. Est ainsi marquée, véritablement, une filiation entre les deux écrivains, avec cependant toutes les nuances nécessaires, Hébert donnant à la parole poétique une inflexion plus subjective, et plus opératoire, que Saint-Denys Garneau.

L'autre contribution un peu roborative est celle de Daniel Marcheix, qui tente une étude comparative, à partir du Journal de Garneau et de deux textes narratifs d'Hébert, des modalités de «l'engagement du masculin dans une aventure perceptive singulière où le regard et la vision sont les vecteurs d'un rapport destructeur au monde, à l'Autre et à soi » (102). Je n'irai pas jusqu'à dire que l'intention est toujours très claire et que la trajectoire de l'analyse se signale par sa netteté. Il demeure néanmoins que la démarche consistant à repérer des similitudes esthésiques entre les mouvements perceptifs garnéliens et ceux des personnages masculins du «Torrent» et d'Un habit de lumière est stimulante. Pour le chercheur de l'Université de Limoges, le sujet du Journal et les protagonistes masculins des fictions étudiées se caractériseraient par une même impuissance visuelle, laquelle déterminerait à son tour un barrage par rapport au réel et donc «un régime de présence placé sous le signe de

$$
++
$$

2 Celui-ci procède, comme le mentionne Nathalie Watteyne dans sa "Présentation », d'une journée d'étude ayant eu lieu à Sherbrooke le 6 mai 2006. 
l'incomplétude et de l'absence au monde» (105). Chez les deux écrivains, l'inaccessibilité du monde réel signerait un échec du masculin, avec des nuances que Marcheix saisit fort bien, même si sa conclusion sacrifie davantage, me semble-t-il, au plaisir de la formule qu'à la contrainte de la limpidité.

On passera rapidement sur la comparaison très empirique que Gisèle Huot établit entre les deux poètes: on a ici affaire à un bref survol, essentiellement redondant par rapport à ce qu'on sait déjà. Je ne dirai qu'un mot, pour terminer, du travail d'Andrée-Anne Giguère sur les usages de l'essai chez Garneau et chez Hébert. Le sujet m'apparaît important et l'étude bien lancée; mais comme le corpus est somme toute assez restreint, surtout chez la seconde, il me semble qu'il faudrait forer plus profond, soit en lisant de plus près encore les essais en cause, soit en creusant plus spécifiquement le rapport (dialectique?) entre écriture essayistique et écriture de la poésie et/ou du roman chez les deux cousins.

Cela dit, je me promets bien, à l'avenir, de suivre plus attentivement la publication des Carnets Anne Hébert. Il faudra voir si c'est là que s'effectuera le travail de fond sur l'œuvre de la disparue - celui, suivi et exigeant, que les revues plus généralistes peuvent difficilement soutenir.

La traduction est l'une des voies par lesquelles les classiques d'une littérature trouvent une nouvelle vie, une nouvelle audience, un nouveau destin, quand ce n'est pas une nouvelle forme qui les révèle un peu plus, ou différemment, au public pour lequel ils avaient été conçus. La traduction thêâtrale, a fortiori, est révélatrice des enjeux de la réception transculturelle des œuvres: destinée à soutenir un art public, immédiat, qui repose sur le partage et la communication in situ, elle constitue l'inscription concrète du trajet que doit accomplir un texte pour passer d'une langue à l'autre, d'une culture à l'autre. Ces enjeux se situent précisément au cœur du livre de Louise Ladouceur, Making the Scene. La traduction du thêatre d'une langue officielle à l'autre au Canada ${ }^{3}$, ouvrage qui, au moyen de la théorie des polysystèmes de l'École de Tel-Aviv, analyse la circulation des textes théâtraux, et accessoirement des productions, entre le Canada francophone et le Canada anglophone depuis la fin des années 1950 jusqu'aux années 1990. On saluera d'entrée de jeu l'ambition théorique de l'ouvrage; celui-ci aspire en effet à mettre en œuvre les propositions théoriques d'Itamar Even-Zohar et surtout de Gideon Toury, qui s'est plus particulièrement penché sur la traduction littéraire. À ce modèle dit "fonctionnaliste», Ladouceur entend au surplus conjoindre une dimension plus individuelle du traduire (17), cette fois en mobilisant les travaux d'Antoine Berman.

Après avoir mis en évidence, dans un très court premier chapitre, les principaux jalons de l'histoire de la traduction littéraire au Canada et fait ressortir la com-

3 Louise Ladouceur, Making the Scene. La traduction du théâtre d'une langue officielle à l'autre au Canada, Québec, Éditions Nota bene, coll. "Sciences humaines/Théâtre», 2005. Avec une préface d'Edward Dickinson Blodgett. 
mande idéologique qui sous-tend cette histoire (représenter l'Autre à travers soi, combler le fossé entre les "deux solitudes»), l'auteure prend très exactement la mesure du corpus global des traductions théâtrales du français vers l'anglais et de l'anglais vers le français, dégageant quelques grandes tendances qui vont influencer la sélection des pièces au fil des décennies. Puis elle détermine, de fort convaincante manière, la composition de son corpus d'analyse, soit douze pièces, six de chaque langue, réparties en six segments temporels (1962-1971, 1972-1974, 1984-1986, 1986-1989, 1990-1991, 1997-1998), choisies dans l'œuvre des dramaturges les plus importants des deux communautés et traduites par les médiateurs les plus actifs. Le troisième chapitre est ensuite consacré à la présentation du cadre théorique et méthodologique. Si la théorie des polysystèmes a pour but «d'observer la fonction de la littérature traduite dans son contexte adoptif et la position qui lui est assignée au sein du polysystème récepteur» (61-62), la critique des traductions de Berman, elle, entend envisager «le sujet traduisant et son rapport conscient ou inconscient au texte et aux enjeux plus personnels dont il est l'objet» (70). Les deux «méthodes » doivent ainsi se compléter, l'une prenant en écharpe ce que l'autre laisse échapper : la dimension individuelle, pour l'une, la dimension plus systémique et normative (c'est-à-dire les normes linguistiques, littéraires et discursives), pour l'autre. Longuement décrit, et repoussant d'autant le début de l'étude proprement dite, le cadre d'analyse est précis, rigoureux, si ce n'est rigide et mécaniste: les douze pièces seront soumises à la même analyse des "données préliminaires", des "macro- et microstructures» et du "contexte systémique», avec ce résultat que l'ensemble paraît parfois légèrement répétitif, voire scolaire. C'est par conséquent ailleurs que l'auteure fait montre de toute la souplesse dont elle est capable: dans la critique des traductions elles-mêmes, dans le relevé de la trajectoire fluctuante qu'accomplit la traduction théâtrale au Québec et au Canada au cours de la période. Si Ladouceur sait se montrer critique à l'endroit des traducteurs, ce n'est toutefois pas toujours le cas en ce qui concerne les théoriciens de la traduction; il me semble qu'elle avalise trop volontiers ce qu'E. D. Blodgett et surtout Annie Brisset (dont les travaux paraissent très historiquement et idéologiquement situés) disent des processus traductionnels en ce pays. Ici, c'est vis-à-vis de la recherche antérieure qu'on aurait aimé que l'analyste prenne davantage ses marques.

Je ne m'attarde pas aux analyses particulières, au demeurant fort instructives, et m'arrête plutôt aux conclusions, essentiellement exposées au chapitre 64 . Du côté anglophone - qui traduit bien davantage que le Québec et qui, ce faisant, se trouve à s'appuyer sur le dynamisme de la jeune dramaturgie québécoise pour promouvoir un nationalisme culturel canadien tout à fait porteur au cours de la décennie du centenaire de la Confédération - , on traduit d'abord les premiers dramaturges québécois (Gélinas, Dubé, Gurik, notamment) dans une optique d'adéquation à l'original, non sans souligner quelques éléments de "québécité» qui accréditent les stéréotypes du Canada anglais à notre endroit. Plus tard, avec Les belles-sœurs, le

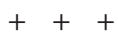

4 Fort succincte heureusement, la conclusion est redondante par rapport aux éléments contenus dans ce sixième chapitre. 
théâtre québécois connaît un succès fulgurant outre-Outaouais, à telle enseigne qu'il en vient à occuper, selon les termes de la théorie des polysystèmes, une position primaire au sein du système anglo-canadien. Malgré cette réussite, la «révolution du joual» ne va pas sans compliquer les choses, commente Louise Ladouceur:

on traduit alors Tremblay dans une langue générique et on accentue le recours aux sacres et aux gallicismes chargés d'attester de [sic] l'inhérente "québécitude » du texte traduit. [...] La critique peut ainsi acclamer un nouveau dramaturge canadien, sans tenir compte de la spécificité de son écriture et de ce qu'elle propose de révolutionnaire. (213)

Louise Ladouceur poursuit:

on peut dire que le Tremblay présenté à Toronto est véritablement canadien puisque la traduction de son œuvre en propose une lecture canadienne, dans laquelle on a évacué la question de la langue et mis l'accent sur une altérité québécoise non menaçante. (213)

Cette appropriation - voire cette expropriation - du thêâtre de Tremblay et des autres dramaturges majeurs des années 1970 se poursuivra jusqu'à ce que les traductrices féministes anglophones commencent à s'intéresser au théâtre des femmes en se proposant de mettre en relief le genre féminin dans la réécriture des textes, et jusqu'à ce que le foyer de la traduction de la dramaturgie québécoise vers l'anglais se déplace à Montréal avec la mise sur pied des programmes de traduction du Centre d'essai des auteurs dramatiques (CEAD). Les auteurs des années 1980 et 1990 - Jovette Marchessault, Normand Chaurette, René-Daniel Dubois, Michel Marc Bouchard, Daniel Danis, Jean-Marc Dalpé, etc. - bénéficieront particulièrement de cette conjoncture dans laquelle « il devient difficile de ne pas tenir compte de la problématique particulière de la langue et de sa primauté au sein d'un système dramaturgique où "[t]he presence of that spoken language $[\ldots]$ is a statement in itself" ${ }^{5}$ " (217). Dans le contexte d'une langue hégémonique et triomphante comme l'anglais, le théâtre tend en effet à ne pas mettre la langue en question, et la scène canadienne a l'habitude d'un idiome naturel, sinon naturaliste; le thêâtre québécois, en revanche - et c'est là une des difficultés de la traduction -, n'a de cesse de s'interroger sur la langue parlée, par le truchement du joual au cours des années 1960 et 1970, par d'autres moyens ensuite, tout aussi problématiques au Canada anglais: l'hyperlittérarisation du dialogue chez Jovette Marchessault, la rupture ironique des registres de langue chez Michel Marc Bouchard, le français sciemment construit de Normand Chaurette, pour ne mentionner que ces trois cas. Avec le changement de la donne au cours des années 1980, ce n'est toutefois plus le contexte récepteur anglophone qui détermine le choix des pièces et les stratégies traductionnelles, mais le contexte source,

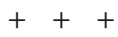

5 La citation dans la citation est de la traductrice montréalaise Linda Gaboriau. 
qui sélectionne les textes qu'il désire promouvoir et diffuser; du coup, les procédés de mise en relief de l'altérité québécoise et les traductions « ethnographiques» tombent en désuétude, fût-ce au risque de décevoir les attentes du public canadien.

Du côté francophone, on traduit d'abord très peu le théâtre canadien-anglais: le vis-à-vis anglophone étant perçu comme menaçant et devant, de ce fait, être tenu à distance, on décide de voir le théâtre canadien à travers le préjugé qui l'assimile à une pâle resucée des dramaturgies américaine et britannique, enlisé dans un naturalisme ennuyeux à périr. Des traductions isolées, voire des adaptations, percent toutefois le marché de loin en loin, Charbonneau et le Chef de John McDonough en étant le meilleur exemple. Conventionnelles, souvent didactiques ou documentaires, les pièces de la jeune dramaturgie anglo-canadienne restent en somme confinées à une position secondaire dans le polysystème québécois. Jusqu'à l'avènement, à partir des années 1990, d'une nouvelle vague thêâtrale plus spectaculaire que spéculaire (40) - avec Brad Fraser, Judith Thompson et George F. Walker - , ce sont surtout des comédies (de David Freeman, de Norm Foster) qui se verront traduites au Québec, dans des versions souvent adaptées où l'on aura gommé le cadre canadienanglais et prolétarisé la langue pour la rendre plus proche du joual alors dominant sur les scènes. Cette tendance à l'adaptation, note Ladouceur, se maintiendra même lorsque cette pratique aura perdu de sa faveur auprès des critiques, et l'insistance à traduire en une langue populaire persistera en dépit du fait qu'elle finit par «tranche[r] avec les esthétiques verbales expérimentées au Québec à la même époque » (224). Tout se passe alors «comme si cette recherche formelle était strictement réservée à la création alors que la traduction demeure soumise à la norme antérieure privilégiant le recours au joual» (224). Les années 1990 vont cependant opérer une rupture: dans les traductions de Fraser et de Walker, on conserve le lieu de l'action (très peu marqué du reste chez Walker) et «on s'éloigne aussi de la langue populaire pour viser une langue familière ou courante» (225). C'est une indication que non seulement les «mœurs» traductionnelles québécoises ont changé, mais que la dramaturgie canadienne-anglaise a atteint un niveau de qualité qui force le respect des traducteurs et qui, du même coup, la fait passer à une situation primaire dans le système québécois. Si les Fraser et Walker influencent des dramaturges tels que François Archambault ou Serge Boucher, en revanche la langue de ces derniers tend à s'imposer dans les traductions qu'on donne de leurs homologues anglophones. De là à dire qu'on assiste à un "rééquilibrage» entre les dramaturgies québécoise et anglo-canadienne, il y a un pas que Louise Ladouceur se garde de franchir; elle constate en effet la persistance d'asymétries entre les corpus et le statut des langues en transaction, et elle montre par ailleurs que si le thêâtre québécois conserve un ancrage assez fort dans sa réalité propre, le thêâtre anglophone doit peut-être une part de son succès à la façon dont il exprime, plutôt qu'une identité canadienne, l'emprise américaine qui s'exerce sur une culture mondialisée. 